\title{
Longitudinal Age-Related Changes in 24-Hour Total Heart Beats and Premature Beats and Their Relationship in Healthy Elderly Subjects
}

\author{
Hirofumi TASAKI, ${ }^{1} \mathrm{MD}$, Takumi Serita, ${ }^{1} \mathrm{MD}$, Chiaki Ueyama, ${ }^{1} \mathrm{MD}$, \\ Kouei Kitano, ${ }^{1} \mathrm{MD}$, Shinji Seto, ${ }^{1} \mathrm{MD}$, Katsusuke Yano, ${ }^{1} \mathrm{MD}$, \\ and A. John CAMM, ${ }^{2} \mathrm{MD}$
}

\begin{abstract}
SUMMARY
The aim of this study was to conduct a longitudinal follow-up on age-related changes in 24-hour total heart beats (THBs) and total premature beats and their correlations in healthy elderly subjects. In 15 healthy elderly subjects (mean age, $70.0 \pm 4.1$, age range at 1st recording, 64 to 80 years, 10 females, 5 males), we conducted Holter monitoring twice at an interval of 15 years and analysed age-related changes in THBs, atrial premature beats (APBs), and ventricular premature beats (VPBs), as well as their correlations.

The results indicated that THBs, APBs, and VPBs all significantly increased with age in the healthy elderly subjects at a mean age of $70.0 \pm 4.1$ (THB: $91074.1 \pm 11515.3$ versus $99457.5 \pm 12131.0 ; P=0.0004$, APB: $119.2 \pm 97.8$ versus $884.4 \pm 1193.8 ; P=0.0008$, VPB: $15.2 \pm 53.6$ versus $140.7 \pm 228.9 ; P=0.0328)$. Moreover, we divided the subjects into increase and nonincrease groups based on the age-related changes in APB and VPB for 15 years ( $[n]$; Inc-APB: Noninc-APB $=6: 9$, Inc-VPB: Noninc-VPB $=5: 10)$. In the increase groups, premature beats tended to increase in proportion to changes in THBs with age (APB: $\mathrm{Y}=207.488+0.136 * \mathrm{X}, r=0.848, P=0.0303$; VPB: $\mathrm{Y}=-27.594+0.028$ $* \mathrm{X}, r=0.727, P=0.1921)$.
\end{abstract}

In conclusion, this 15 -year follow-up of Holter recordings in healthy elderly subjects revealed that THBs, APBs, and VPBs increased with age, and that the increases in premature beats, especially APBs, were in proportion to those in THBs. (Int Heart J 2006; 47: 549-563)

Key words: 24-hour total heart beat, Atrial premature beat, Ventricular premature beat, Healthy elderly subject, Age-related change

IN general, in healthy adults younger than 70 years old, maximum heart rate (HR) on exercise, maximum and mean HR during Holter monitoring, and intrinsic HR after pharmacological autonomic block are known to decrease with age. ${ }^{1-4)}$

From ${ }^{1}$ Cardiovascular Medicine, Course of Medical and Dental Sciences, Graduate School of Biomedical Sciences, Nagasaki University, Nagasaki, Japan, ${ }^{2}$ Cardiological Sciences, Cardiovascular Medicine, St. George's Hospital Medical School, University of London, London, United Kingdom.

Address for correspondence: Hirofumi Tasaki, MD, Cardiovascular Medicine, Course of Medical and Dental Sciences, Graduate School of Biomedical Sciences, Nagasaki University, Sakamoto 1-7-1, Nagasaki 852-8501, Japan.

Received for publication January 11, 2006.

Revised and accepted April 10, 2006. 
On the other hand, it has been reported that there is a greater prevalence of premature beats in healthy elderly people than in healthy younger adults, and that the frequency as well as the prevalence of arrhythmia increases with age. ${ }^{5,6)}$ There are many studies on the relationship between age and HR or premature beats, especially ventricular premature beats (VPBs). ${ }^{7,8)}$ However, most of the reports are based on cross-sectional studies and there are few long-term follow-up studies on the findings of Holter monitoring in the elderly, ${ }^{9-12)}$ especially on HR and frequency of premature beats. Accordingly, the longitudinal changes in HR and premature beats with aging remain to be elucidated, especially in the elderly.

Moreover, some reports have shown that ventricular or atrial arrhythmias have demonstrated a circadian rhythm in parallel with HR. ${ }^{13-15)}$ However, few studies have revealed the relationships between age-related changes in HR and those in premature beats. The analysis of the relationships may provide new insights into the age-related changes in HR as well as premature beats.

Previously, we conducted a longitudinal HR study over 15 years by means of Holter monitoring in healthy elderly subjects and reported that HR in the morning and in the afternoon increased with age, unlike the previous reports in healthy adults younger than $70 .{ }^{16)}$ Then, we assessed the age-related changes in 24-hour total heart beats (THBs) and those in 24-hour total premature beats over 15 years and the relationships in the healthy elderly subjects in whom premature beats were thought to increase with age.

\section{METHODS}

Study population and Holter monitoring (Figure 1): The methods were those described in detail in a previous study, ${ }^{16)}$ with some additions as follows. Among 164 healthy subjects (age range, 14 to 87 , mean age, $54.2 \pm 21.3$ years, at first monitoring; 90 females and 74 males) in whom we conducted the first Holter monitoring, ${ }^{18)}$ there were 89 healthy subjects older than 60 (age range, 61 to 87 , mean, $72.0 \pm 4.9$ years, at first monitoring; 58 females and 31 males). The 89 healthy elderly subjects had been undergoing community-based annual health checks as often as possible, including physical examination, electrocardiography, chest x-rays, a complete blood count, and blood biochemistry tests. Among these 89 elderly subjects, we were able to confirm the medical history in 74 by telephone 15 years after the first monitoring. Forty-eight elderly subjects were still alive and 26 had died (Figure 1, Step I).

We enrolled only those subjects who did not have any present illness or history of heart diseases. We thus were able to conduct a second Holter monitoring in 19 subjects from whom we obtained informed consent (Figure 1, Step II). We then verified again that they did not have any heart diseases or were taking any 


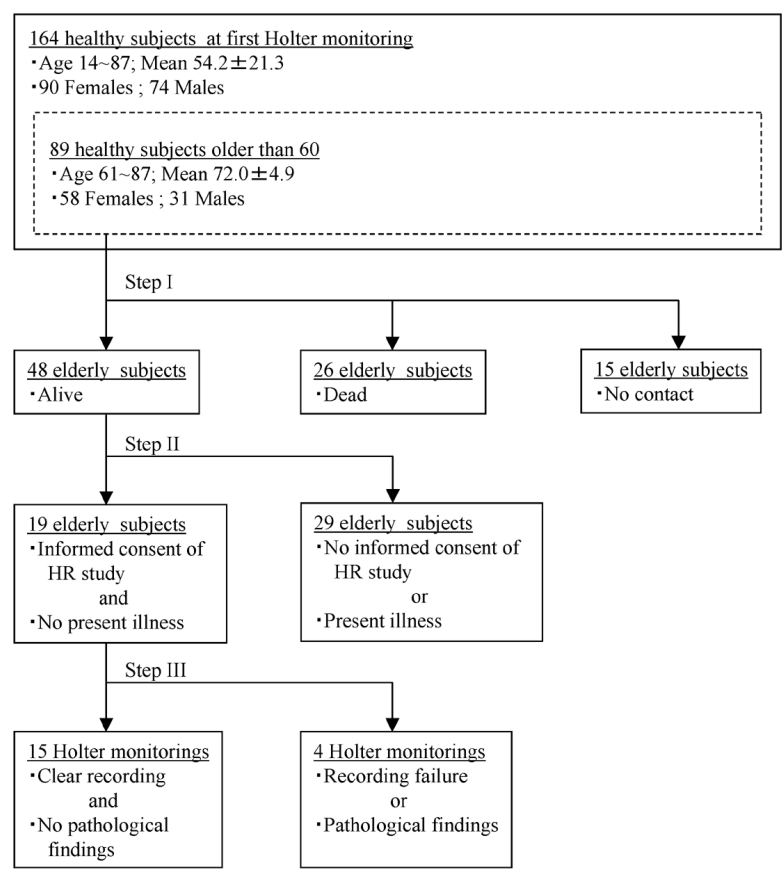

Figure 1. Flowchart of entry process for 15 healthy elderly subjects 15 years later. The subjects were classified through step I and step II based on the information verified on the phone or in person after a 15 -year interval. The 15 subjects were then enrolled in step III according to the recording conditions and findings of the 2nd Holter monitoring. HR indicates heart rate.

drugs which could affect their heart rate and/or rhythm, when conducting the second Holter monitoring. We excluded subjects whose Holter recording revealed recording failure or a pathological finding. As a result, there were only 15 subjects whose second Holter recordings were clear enough to be analyzed properly and precisely (Figure 1, Step III). We examined the first and second Holter monitorings in the 15 healthy elderly subjects, whose daily physical activities were typical for their ages. They had all lived in the countryside in Japan and were capable of enjoying a short walk, a game of Japanese gateball (croquet-like game played by seniors), or light farmwork like gardening even 15 years later. Among them, there were 13 nonsmokers, 1 confirmed smoker, and 1 unknown.

Data analysis: Every Holter monitoring was of sufficient quality for an analysis of THBs, atrial premature beats (APBs), and VPBs. The first and second mean Holter monitoring times were 22 hours and 52 minutes and 23 hours and 48 minutes, respectively. They were all "corrected" or revised to 24 hours. We calculated 
and assessed the changes in THBs, APBs, and VPBs over 15 years, as well as the correlations among them.

Statistics: (I) Comparison between 1st and 2nd monitoring periods: First, we tested the normal distribution of subjects using the Kolmogorov test. If a normal distribution was observed, we used the paired $t$ test to compare the first and second recordings. The Wilcoxon test was used if a normal distribution was not observed. (II) Comparison between increase and nonincrease groups of premature beats: First, we tested the normal distribution and equal variance of subjects using the Kolmogorov test and F test, respectively. If both statistical conditions were met, the unpaired $t$ test was used to compare the increase and nonincrease groups. If neither condition was met, the Mann-Whitney test was used. (III) Test of correlation: Pearson's correlation coefficient was used visually to test for correlations. However, if the distribution of the subjects deviated to a great extent, Spearman's correlation coefficient was used. A $P<0.05$ was considered to be significant.

\section{RESULTS}

Changes in total heart beats and premature beats with age in healthy elderly subjects:

a) Changes in total heart beats with age.

THBs had significantly increased after 15 years (Table I). THBs in 13 of the 15 healthy elderly subjects had increased, while those in the other 2 subjects decreased (Figure 2).

Table I. Changes in THBs, Total APBs and Total VPBs Over 15 Years in Healthy Elderly Subjects

\begin{tabular}{lrccc}
\hline & $n$ & 1st record & 15 years later & $P$ \\
\hline THB & 15 & $91074.1 \pm 11515.3$ & $99457.5 \pm 12131.0$ & $0.0004^{*}$ \\
Inc-group & 13 & $91632.6 \pm 12272.9$ & $101586.0 \pm 11461.7$ & - \\
Noninc-group & 2 & $87443.4 \pm 4304.0$ & $85621.8 \pm 6484.4$ & - \\
APB & 15 & $119.2 \pm 97.8$ & $884.4 \pm 1193.8$ & $0.0008^{* *}$ \\
Inc-group & 6 & $183.5 \pm 91.1$ & $1972.5 \pm 1264.2$ & $0.0277^{* *}$ \\
Noninc-group & 9 & $76.3 \pm 80.0^{*}$ & $159.0 \pm 123.4^{*}$ & $0.0024^{*}$ \\
VPB & 15 & $15.2 \pm 53.6$ & $140.7 \pm 228.9$ & $0.0328^{*}$ \\
Inc-group & 5 & $42.4 \pm 93.1$ & $418.0 \pm 357.9$ & $0.0244^{*}$ \\
Noninc-group & 10 & $1.6 \pm 1.4^{\dagger}$ & $2.1 \pm 1.5^{*}$ & $0.2606^{*}$ \\
\hline
\end{tabular}

* paired $t$-test $\& * *$ Wilcoxon test, comparison of 1 st record with 15 years later ; \# two sample $t$-test: $P=0.0158 \&$ q Mann-Whitney test: $P=0.0015$, compared with Inc-APB group ; $\dagger \& \ddagger$ Mann-Whitney test: $P=0.7518 \& P=$ 0.0020 , respectively, compared with Inc-VPB group ; Values are mean \pm SD. 


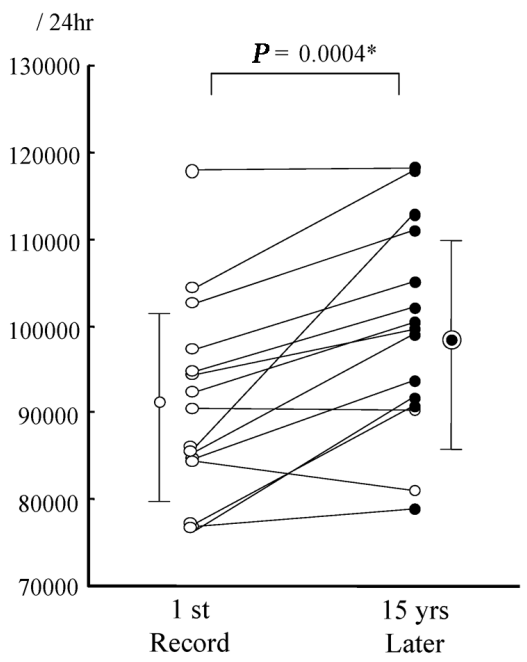

Figure 2. Changes in THBs over 15 years in healthy elderly subjects.

The 13 closed circles of 15 years later indicate the Inc-group of THB, while the 2 open circles indicate the Noninc-group of THB. * paired $t$ test.
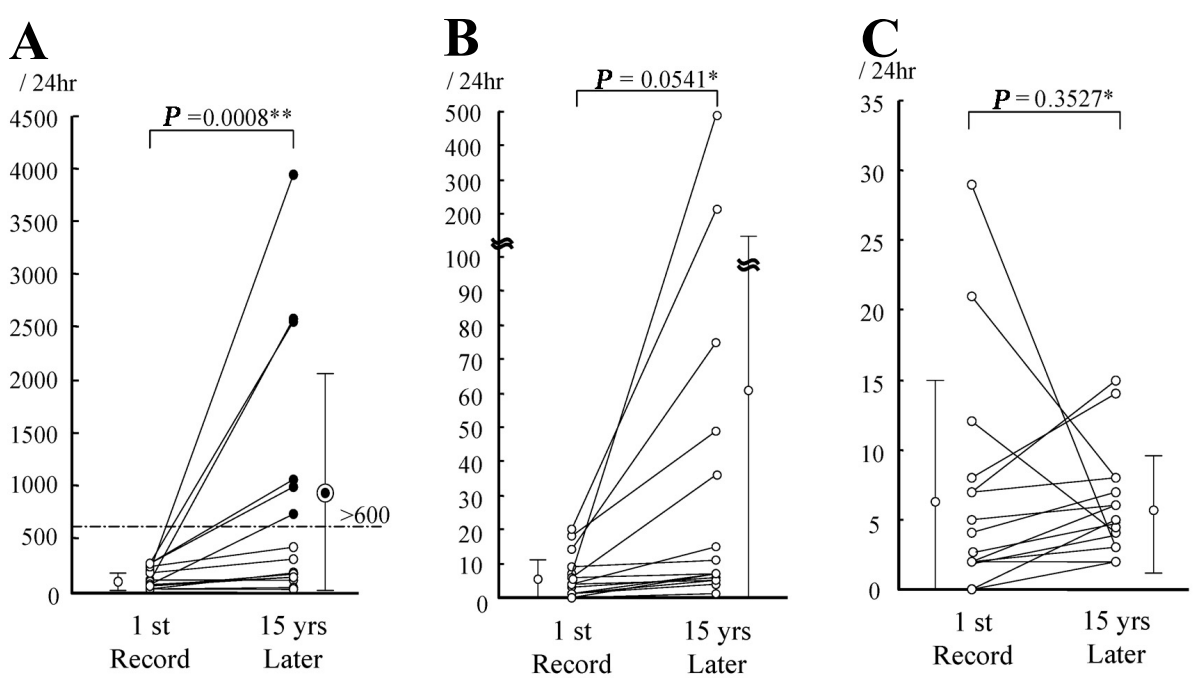

Figure 3. Changes in APBs over 15 years in healthy elderly subjects.

(A) Total APBs: The 6 closed circles of 15 years later indicate the Inc-APB group in which there were obvious and significant increases in 24-hour total APBs, while the 9 open circles indicate the Noninc-APB group in which there were slight, although significant, increases in 24-hour total APBs. (B) Total APB runs, (C) APB maximum run, * paired $t$ test, ** Wilcoxon test. 


\section{b) Changes in atrial premature beats with age.}

The number of APBs at the first Holter monitoring in the healthy elderly subjects was less than 300/day. The APBs in this population increased significantly after 15 years (Table I, Figure 3A). Given that the APB increase group (Inc-APB group) refers to the group in which APBs increased more than 3 times the count at first monitoring and up to more than 600/day 15 years later, and that the APB nonincrease group (Noninc-APB group) refers to the group in which APBs increased less than 3-fold or up to less than 600/day 15 years later, the 15 healthy elderly subjects were ultimately divided into the following 2 groups; IncAPB group $(n=6)$ in which APBs increased up to 725 to 3949/day and NonincAPB group $(n=9)$ in which APBs increased up to 410/day or less (Figure 3A). APBs in the Inc-APB and Noninc-APB groups had increased significantly 15 years later (Table I, Figure 3A). In addition, APBs in the Inc-APB group were significantly higher than those in the Noninc-APB group at the first monitoring as well as the second monitoring (Table I).

Moreover, total APB runs of couplets or salvos tended to increase with age (Figure 3B). However, all subjects with maximum APB runs of more than 10 suc-

A

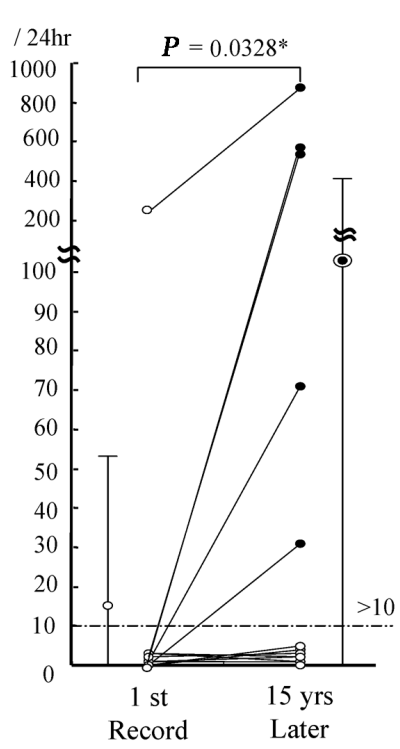

B

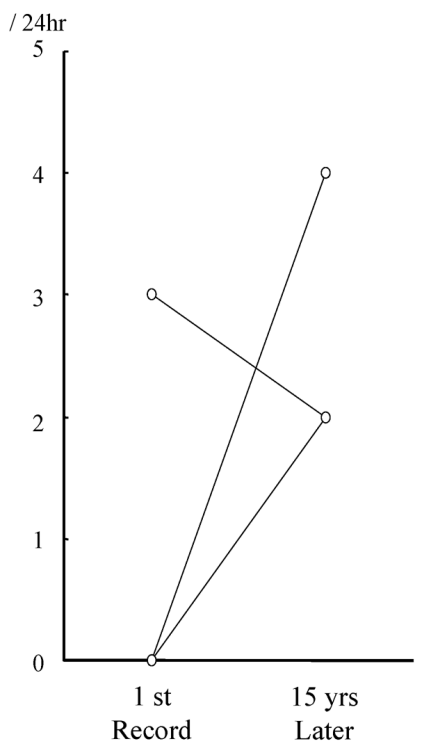

Figure 4. Changes in VPBs over 15 years in healthy elderly subjects.

A: Total VPBs: The 5 closed circles of 15 years later indicate the Inc-VPB group in which there were significant increases in 24-hour total VPBs, while the 10 open circles indicate the Noninc-VPB group in which there were no changes in 24-hour total VPBs. B: VPB couplets: There were 3 VPB couplets in one subject at first recording, while there were 4 couplets in one subject and 2 couplets in 2 subjects 15 years later. * paired $t$ test. 
cessive beats at first monitoring had maximum APB runs of less than 10 successive beats 15 years later (Figure 3C).

c) Changes in ventricular premature beats with age.

VPBs in the healthy elderly subjects in this study were not as frequent as APBs, and except for 1 subject (VPBs: 209/day), were 5 or less at first monitoring (Table I, Figure 4A). VPBs in this population as a whole had increased significantly 15 years later. Given the VPB increase group (Inc-VPB group) refers to the group in which VPBs increased more than 3 times over the first monitoring and up to more than 10/day 15 years later, and that the VPB nonincrease group (Noninc-VPB group) refers to the group in which VPBs increased less than 3-fold or less than 10/day 15 years later, the 15 healthy elderly subjects were also divided into the following 2 groups; an Inc-VPB group $(n=5)$ in which VPBs increased up to 31 to 866/day and a Noninc-VPB group $(n=10)$ in which VPBs remained at 5/day or less (Figure 4A). Although the number of VPBs of 5 elderly subjects in the Inc-VPB group increased significantly 15 years later, those of 10 elderly subjects in the Noninc-VPB group did not change (Table I, Figure 4A). Unlike
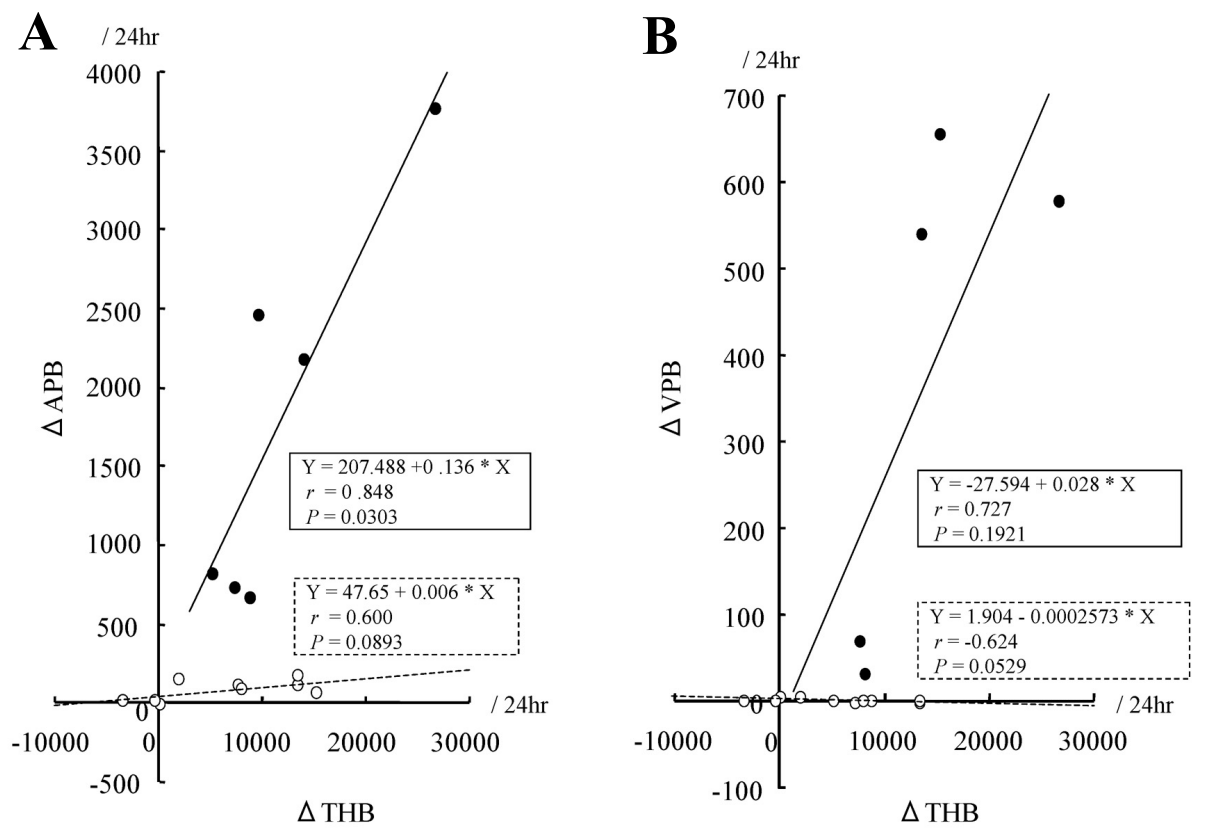

Figure 5. Correlations between $\triangle \mathrm{THBs}$ and $\triangle \mathrm{APBs}$ or $\triangle \mathrm{VPBs}$ over 15 years in healthy elderly subjects.

A: Correlation between $\triangle \mathrm{THBs}$ and $\triangle \mathrm{APBs}$ : the 6 closed circles indicate the Inc-APB group, in which $\triangle \mathrm{APB}$ was correlated significantly with $\triangle \mathrm{THB}$, while in the Noninc-APB group composed of the 9 open circles, there was no significant correlation between $\triangle \mathrm{THB}$ and $\triangle \mathrm{APB}$. B: Correlation between $\triangle \mathrm{THB}$ and $\triangle \mathrm{VPBs}$ : The 5 closed and 10 open circles indicate the Inc-VPB group and the Noninc-VPB group, respectively, in which there was no significant correlation between $\triangle \mathrm{THB}$ and $\triangle \mathrm{VPB}$. The solid and broken lines indicate the regression lines in the Inc-group and Noninc-group, respectively. 
APB, there was no significant difference in the number of VPBs between the IncVPB and Noninc-VPB groups at first monitoring (Table I).

In addition, in the VPB runs, there were 3 VPB couplets in one subject at first monitoring, while there were $4 \mathrm{VPB}$ couplets in one subject and 2 couplets in 2 subjects 15 years later, although there were no more successive beats at the first or second monitorings (Figure 4B).

Relationship between changes in total heart beats and premature beats with age in healthy elderly subjects: We assessed the relationship between changes in THB $(\triangle \mathrm{THB}), \mathrm{APB}(\triangle \mathrm{APB})$, and VPB $(\triangle \mathrm{VPB})$ over 15 years in healthy elderly subjects.

a) Relationship between $\triangle T H B$ s and $\triangle A P B$ s with age (Figure 5A).

$\triangle \mathrm{THB}$ and $\triangle \mathrm{APB}$ in the Inc-APB group were significantly positively correlated. On the other hand, $\triangle \mathrm{THB}$ and $\triangle \mathrm{APB}$ were slightly correlated in the Noninc-APB group, although the correlation was not significant.

b) Relationship between $\triangle T H B$ s and $\triangle V P B s$ with age (Figure 5B).

There was a slight positive correlation between $\triangle \mathrm{THB}$ and $\triangle \mathrm{VPB}$ in the IncVPB group and a slight inverse correlation between $\triangle \mathrm{THB}$ and $\triangle \mathrm{VPB}$ in the Noninc-VPB group in this population. However, neither correlation was significant. c) Relationship between $\triangle A P B s$ and $\triangle V P B s$ with age (Figure 6).

We tested the relationship between $\triangle \mathrm{APB}$ and $\triangle \mathrm{VPB}$ using Spearman's correlation coefficient because of the deviation in the distribution. The results indicated there was no significant correlation between the 2 .

Relationships between ages at first monitoring and changes in total heart beats and premature beats over 15 years (Table II): We examined the differences in age at

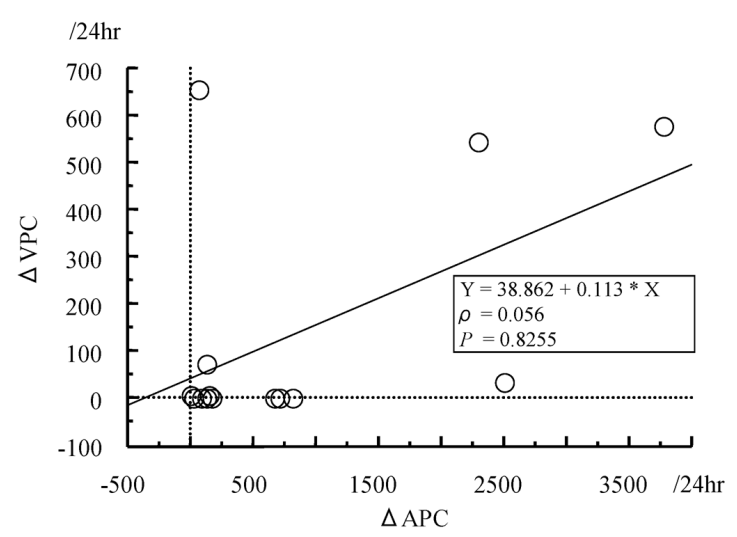

Figure 6. Correlation between $\triangle \mathrm{APBs}$ and $\triangle \mathrm{VPBs}$ over 15 years in healthy elderly subjects.

$\rho$ indicates Spearman's correlation coefficient. 
Table II. Ages at First Monitoring and Changes in THBs, APBs and VPBs Over 15 Years in Healthy Elderly Subjects

\begin{tabular}{|c|c|c|c|}
\hline THB & Inc-group & Noninc-group & $P$ \\
\hline \multirow{3}{*}{$\begin{array}{l}\quad n \\
\text { Age at } 1 \text { st record } \\
\text { Correlation between age at } 1 \text { st record and } \triangle \mathrm{THB} \\
\quad \text { in increase group }\end{array}$} & 13 & 2 & \\
\hline & $70.5 \pm 4.0$ & $70.0 \pm 7.1$ & - \\
\hline & $\left(\begin{array}{rl}\mathrm{Y} & =5797+59 \times \mathrm{X} \\
r & =0.035 \\
P & =0.9108\end{array}\right.$ & & \\
\hline APB & Inc-group & Noninc-group & $P$ \\
\hline \multirow{3}{*}{$\begin{array}{l}\quad n \\
\text { Age at } 1 \text { st record } \\
\text { Correlation between age at 1st record and } \triangle \mathrm{APB} \\
\text { in increase group }\end{array}$} & 6 & 9 & \\
\hline & $71.2 \pm 3.1$ & $69.9 \pm 4.8$ & $0.2878 \#$ \\
\hline & $\left(\begin{array}{rl}\mathrm{Y} & =9639-110 \times \mathrm{X} \\
r & =-0.266 \\
P & =0.6379\end{array}\right.$ & & \\
\hline VPB & Inc-group & Noninc-group & $P$ \\
\hline \multirow{3}{*}{$\begin{array}{l}\quad n \\
\text { Age at } 1 \text { st record } \\
\text { Correlation between age at } 1 \text { st record and } \triangle \mathrm{VPB} \\
\text { in increase group }\end{array}$} & 5 & 10 & \\
\hline & $69.8 \pm 2.9$ & $70.7 \pm 4.7$ & $0.3527 \#$ \\
\hline & $\left(\begin{array}{rl}\mathrm{Y} & =5116-68 \times \mathrm{X} \\
r & =-0.648 \\
P & =0.2747\end{array}\right.$ & & \\
\hline
\end{tabular}

\# two sample $t$-test, comparison of Inc-group with Noninc-group; $r$ is Peason's correlation coefficient; Values are mean \pm SD.

first monitoring between the THB, APB, and VPB increase and nonincrease groups. There was no statistically significant difference in age between the APB or VPB increase and nonincrease groups at first monitoring. As there were only 2 subjects in the THB nonincrease group, they could not be compared to the THB increase group or statistically tested with respect to the difference in age between the 2 groups. However, there seemed to be no difference in age between the 2 .

Moreover, the correlations between the ages at first monitoring and the $\triangle \mathrm{THB}, \triangle \mathrm{APB}$ or $\triangle \mathrm{VPB}$ over 15 years in each increase group were tested crosssectionally. Analysis showed that there was no correlation in either relationship.

\section{DisCUSSION}

Changes in total heart beats and premature beats with age in healthy elderly subjects:

a) Changes in total heart beats with age.

Previous cross-sectional HR studies in healthy adults showed that maximal HR on exercise, maximal and mean HR during Holter monitoring, and intrinsic HR after pharmacological autonomic block all decreased with age. ${ }^{1-4)}$ On the con- 
trary, our longitudinal study of 15 healthy elderly subjects at the mean age of 70 showed that the number of THBs was significantly increased 15 years later in most of the subjects (13 of 15 subjects).

The increase in THB with age in healthy elderly subjects may derive from the decrease in cardiac output and total blood volume and respiratory dysfunction with aging, as well as from age-related changes in norepinephrine metabolism at the neuroeffector gaps in the sinus node and norepinephrine spillover from the synapses in heart and other organs to circulatory blood with age, ${ }^{6,16,19,20)}$ or from a decline in vagal activity due to a decrease in nitric oxide and muscarinic receptors with age. ${ }^{21-23)}$ If this HR change was due to the former, that is, cardiopulmonary dysfunction with age, it would increase more in the daytime than in the nighttime. However, the increases in HRs with age in the daytime have been shown to be comparable to those in the nighttime, as our previous study on the same subject showed. ${ }^{16,17)}$ Accordingly, the cause of the increases in HRs in this study might be a factor, such as metabolism, which is not affected by daily activities, but is affected by aging to the same extent in the daytime as in the nighttime.

\section{b) Changes in atrial premature beats with age.}

There have been few studies on the longitudinal age-related changes in APBs. ${ }^{18)}$ In this study, the numbers of APBs in all healthy elderly subjects were less than 300/day at first monitoring, which was within normal limits, and had increased significantly in most 15 years later.

There was no difference in age between the Inc-APB group and the NonincAPB group. The numbers of APBs in both groups increased significantly for the 15 years, although those in the Noninc-APB group increased only slightly, as shown in Table I. Thus, the findings suggest that the number of APBs in $40 \%$ of the healthy elderly people whose APBs were within the normal limit at age 70 increased remarkably thereafter with aging, and that the number of APBs in the rest of the elderly people with a normal frequency of APBs at age 70 increased only slightly. Although the gender differences must be taken into consideration, these results are interesting when the mechanism or background of the increase in APBs with age is considered, and possibly when we predict paroxysmal atrial fibrillation as well.

Moreover, APBs in the Inc-APB group were already significantly higher than those in the Noninc-APB group at the age of 70. Therefore, in healthy elderly subjects at age 70 , there may already be a significant difference in the number of APBs between the 2 groups in which APBs would increase remarkably thereafter and in which APBs would increase only slightly.

Overall, a slight increase in total APB runs 15 years later was observed. If there were more subjects in the study, the increase may have been significant. On the other hand, no clear trend for maximum APB runs in individuals with respect 
to age were seen in our study.

\section{c) Changes in ventricular premature beats with age.}

The increases in VPBs were not as marked as those in APBs for 15 years. VPBs in the Inc-VPB group increased significantly over 15 years, while VPBs in the Noninc-VPB group did not increase. These results seem to indicate that VPBs in approximately $30 \%$ of the healthy elderly whose VPBs were within normal limits at age 70 increased significantly with aging while VPBs in the remaining healthy elderly subjects remained unchanged.

Relationship between changes in total heart beats and premature beats with age in healthy elderly subjects: APBs increased significantly with age not only in the Inc-APB group, but also in the Noninc-APB group among the healthy elderly subjects, as mentioned above. In the Inc-APB group, $\triangle \mathrm{APBs}$ and $\triangle \mathrm{THBs}$ with age were significantly positively correlated. In the Noninc-APB group, the correlation between the 2 was slightly, although not significantly, positive. These results suggest that in $40 \%$ of the healthy elderly people with a normal frequency of APB at age 70, APB increased in proportion to $\triangle \mathrm{THB}$ after 70 years old, while in the remainder, APB exhibited only a slight increase with age but no correlation with $\triangle \mathrm{THB}$.

In the Inc-VPB group, which accounted for about $30 \%$ of the healthy elderly subjects, there was a significant increase in VPBs which showed a positively correlated tendency, although not statistically significant correlation, with $\triangle \mathrm{THB}$ with age, whereas in the Noninc-VPB group, in other words, in the remaining healthy elderly subjects, VPB did not increase 15 years later regardless of the $\Delta \mathrm{THB}$.

Ages at first monitoring and changes in total heart beats and premature beats over 15 years: In this study, we divided the healtly elderly subjects into the increase group and the nonincrease group based on the increase in APB, VPB or THB over 15 years. Thus, to confirm whether or not there were any differences in age between the 2 groups, we compared the ages between the 2 at first monitoring. We found no significant difference in ages between the 2 groups of THB, APB or VPB. Moreover, in each increase group we also assessed with respect to the correlation between age at first monitoring and $\triangle \mathrm{THB}, \triangle \mathrm{APB}$ or $\triangle \mathrm{VPB}$ over 15 years. No significant correlation was found between them. Therefore, the differences in $\triangle \mathrm{THB}, \triangle \mathrm{APB}$, and $\triangle \mathrm{VPB}$ between the increase and nonincrease groups in this study were not derived from the differences in ages of the subjects.

Accordingly, the changes in premature beats, especially APB, which were suggested to increase with age in previous studies, ${ }^{5,6)}$ were much more closely correlated with $\triangle \mathrm{THB}$ over 15 years than with the ages of the subjects at first monitoring. This may be because the observation period in this study was so long that the age-related changes were enhanced all the more. In addition, the reason 
why the increases in premature beats in the increase groups did not correlate with the ages in this study may be because the subject population in this study was so small that the individual dispersion, ie, individual variation, had more impact on the changes in premature beats than did the individual age.

Study implications: Previous HR studies have shown that HR decreases with age in healthy adults. However, there are few reports on the longitudinal age-related changes in HR or THB in healthy elderly subjects, especially those older than 70 years.

In this follow-up after 15 years with Holter monitoring in healthy elderly subjects, THBs, total APBs, and total VPBs all increased significantly with aging. THB and total premature beats in our study were within normal limits at the mean age of 70 at first monitoring. Thus, $\triangle \mathrm{THB}, \triangle \mathrm{APB}$, and $\triangle \mathrm{VPB}$ over 15 years may reflect a kind of aging phenomena of the cardiovascular system that is limited to healthy elderly subjects older than 70. Moreover, although THBs in most subjects in our study increased with age, both premature beats were divided into increase and nonincrease groups 15 years later based on our criteria. In the increase group of premature beats, slight correlations between the increases in premature beats and $\triangle \mathrm{THB}$ were observed, especially $\triangle \mathrm{APB}$ and $\triangle \mathrm{THB}$.

$\mathrm{HR}$ in Holter monitoring is considered to be a well reproducible index. ${ }^{13)}$ Thus, from the findings of this study, given that increases in THB with aging are common and universal phenomena in subjects older than 70 years without any history of heart diseases, it follows that, in a certain percent of the healthy elderly population, premature beats, especially APBs, increase in proportion to $\triangle \mathrm{THB}$, but not THB or HR itself, whereas in the rest of the healthy elderly population, APB increases only slightly while VPB does not increase with age, regardless of $\triangle \mathrm{THB}$.

There have been few reports on the relationship between HR and APBs. Frost, et al reported that low vagal tone and APCs predicted atrial fibrillation and flutter after coronary bypass grafting. ${ }^{24)}$ Bettoni, et al suggested that changes in autonomic nervous activities were associated with the occurrence of atrial fibrillation. ${ }^{25)}$ On the other hand, Pitzalis, et $a l^{7)}$ and Coumel $^{8)}$ reported tachycardiainduced VPC and pointed out the possibility of involvement of sympathetic activities. Moreover, Fleg, et al and Pfeifer, et al described an age-related increase in plasma norepinephrine in elderly subjects at maximum exercise and in the supine position, respectively. ${ }^{19,20)}$ Lakatta attributed this phenomenon to increasing spillover with age from nerve endings into the circulation due to decreased norepinephrine reuptake at the nerve ending, ie, synapse. ${ }^{6}$ In addition, muscle sympathetic nerve activities are also considered to increase with age. ${ }^{26)}$

Given the increases in THB and premature beats in our study were also attributed to age-related changes in autonomic nervous activity or its balance, it 
follows that THB approached a minimum around 70 years of age, while premature beats were on baseline until 70, and that both of them increased thereafter as the age-related changes in autonomic nervous activity were gradually manifested. Thus, in this study, in which we followed-up healthy elderly subjects for 15 years starting at the mean age of 70, we might have observed age-related changes in autonomic nervous activity from the point of view of HR and premature beats.

However, $\triangle \mathrm{APBs}$ and $\triangle \mathrm{VPBs}$ over 15 years were not correlated with each other, suggesting differences in the innervation of or response to autonomic nerves between the atrium and ventricle, ${ }^{27,28)}$ or alternatively, other kinds of agerelated changes in signal transduction through cell membranes or within cells at the foci of arrhythmias. ${ }^{29)}$ In other words, while sharing a common background such as an increase in sympathetic activity and other age-related changes as well as genetic factors in heart, the age-associated changes in APB and VPB might be related to other independent factors peculiar to each arrhythmia or each ventricle. Conclusion: In this investigation, we conducted a longitudinal study in healthy elderly subjects by means of Holter monitoring at an interval of 15 years, assessed the changes in THB and premature beats with age, and then attempted to identify any correlations.

THB was found to increase with aging in most of the healthy elderly subjects studied. As for premature beats, we were able to divide the healthy elderly subjects into 2 groups based on the changes in premature beats with aging, ie, the premature beat increase and nonincrease groups. In the increase group, the increases in premature beats, especially those of APBs, tended to be positively correlated to the increases in THBs. Accordingly, it is possible that the increase in premature beats, generally observed in the elderly, increased in proportion to the increase in THB with age.

These findings might be characteristic of the elderly older than 70 and, at the same time, suggest the mechanism as well as background of various arrhythmias showing increasing frequency with age in the healthy elderly, including sympathovagal balance, age-related degeneration, and genetic factors in heart.

\section{ACKNOWLEDGMENTS}

This work was partly supported by the Fukuda Foundation for Medical Technology, Tokyo, Japan, The Daiwa Anglo-Japanese Foundation, London, UK, and the British Heart Foundation, London, UK. 


\section{REFERENCES}

1. Tanaka H, Monahan KD, Seals DR. Age-predicted maximal heart rate revisited. J Am Coll Cardiol 2001; 37 : 153-6.

2. Kostis JB, Moreyra AE, Amendo MT, Di Pietro J, Cosgrove N, Kuo PT. The effect of age on heart rate in subjects free of heart disease. Studies by ambulatory electrocardiography and maximal exercise stress test. Circulation 1982; 65: 141-5.

3. Umetani K, Singer DH, McCraty R, Atkinson M. Twenty-four hour time domain heart rate variability and heart rate: relations to age and gender over nine decades. J Am Coll Cardiol 1998; 31: 593-601.

4. Jose $\mathrm{AD}$, Collison $\mathrm{D}$. The normal range and determinants of the intrinsic heart rate in man. Cardiovasc Res 1970; 4: 160-7.

5. Fleg JL. Electrocardiographic findings in older persons without clinical heart disease; in Aronow WS 2nd eds: Cardiovascular Disease in the Elderly Patient - Fundamental and Clinical Cardiology, V 36. New York, Decker Communications, 1999: 43-57.

6. Lakatta EG, Levy D. Arterial and cardiac aging: major shareholders in cardiovascular disease enterprises: Part II: the aging heart in health: links to heart disease. Circulation 2003; 107: 346-54. (Review)

7. Coumel P. Rate dependence and adrenergic dependence of arrhythmias. Am J Cardiol 1989; 64: 41J-5.

8. Pitzalis MV, Mastropasqua F, Massari F, et al. Dependency of premature ventricular contractions on heart rate. Am Heart J 1997; 133: 153-61.

9. Camm AJ, Evans KE, Ward DE, Martin A. The rhythm of the heart in active elderly subjects. Am Heart J 1980; 99: 598-603.

10. Martin A, Benbow LJ, Butrous GS, Leach C, Camm AJ. Five-year follow-up of 101 elderly subjects by means of long-term ambulatory cardiac monitoring. Eur Heart J 1984; 5: 592-6.

11. Fleg JL, Kennedy HL. Cardiac arrhythmias in a healthy elderly population: detection by 24-hour ambulatory electrocardiography. Chest 1982; 81: 302-7.

12. Fleg JL, Kennedy HL. Long-term prognostic significance of ambulatory electro-cardiographic findings in apparently healthy subjects greater than or equal to 60 years of age. Am J Cardiol 1992; 70: 748-51.

13. Lanza GA, Cortellessa MC, Rebuzzi AG, et al. Reproducibility in circadian rhythm of ventricular premature complexes. Am J Cardiol 1990; 66: 1099-106.

14. Hayashi H, Fujiki A, Tani M, et al. Circadian variation of idiopathic ventricular tachycardia originating from right ventricular outflow tract. Am J Cardiol 1999; 84: 99-101, A8.

15. Rossi A, Sforza C, Carandente F. Heart rate and extrasystolic arrhythmias in active subjects aged over 90 . A chronobiological study. Chronobiologia 1986; 13: 309-18.

16. Tasaki H, Serita T, Irita A, et al. A 15-year longitudinal follow-up study of heart rate and heart rate variability in healthy elderly persons. J Gerontol A Biol Sci Med Sci 2000; 55: M744-9.

17. Tasaki H, Serita T, Ueyama C, et al. Long-term follow-up of heart rate, heart rate variability and its circadian rhythm in healthy elderly subjects. Circ J 2006; 70: 889-95.

18. Hashiba K. Arrhythmias in the elderly. Jpn J Geriat (Nippon Ronen Igakkai Zasshi) 1989; 26: 101-10. (Japanese)

19. Fleg JL, Tzankoff SP, Lakatta EG. Age-related augmentation of plasma catecholamines during dynamic exercise in healthy males. J Appl Physiol 1985; 59: 1033-9.

20. Pfeifer MA, Weinberg CR, Cook D, Best JD, Reenan A, Halter JB. Differential changes of autonomic nervous system function with age in man. Am J Med 1983; 75: 249-58.

21. Conlon K, Kidd C. Neuronal nitric oxide facilitates vagal chronotropic and dromotropic actions on the heart. J Auton Nerv Syst 1999; 75: 136-46.

22. Massion PB, Balligand JL. Modulation of cardiac contraction, relaxation and rate by the endothelial nitric oxide synthase (eNOS): lessons from genetically modified mice. J Physiol 2003; 546: 63-75. (Review)

23. Brodde $\mathrm{OE}$, Konschak U, Becker K, et al. Cardiac muscarinic receptors decrease with age. In vitro and in vivo studies. J Clin Invest 1998; 101: 471-8.

24. Frost L, Molgaard H, Christiansen EH, Jacobsen CJ, Allermand H, Thomsen PE. Low vagal tone and supraventricular ectopic activity predict atrial fibrillation and flutter after coronary artery bypass grafting. Eur Heart J 1995; 16: 825-31. 
25. Bettoni M, Zimmermann M. Autonomic tone variations before the onset of paroxysmal atrial fibrillation. Circulation 2002; 105: 2753-9.

26. Seals DR, Esler MD. Human ageing and the sympathoadrenal system. J Physiol 2000; 528: 407-17.

27. Cooper T. Terminal innervation of the heart. In Randall E, eds. Nervous Control of the Heart. Baltimore: Williams \& Wilkins; 1965: 130-53.

28. Roskoski R Jr, Mayer HE, Schmid PG. Choline acetyltransferase activity in guinea-pig heart in vitro. J Neurochem 1974; 23: 1197-200.

29. Lakatta EG. Arterial and cardiac aging: major shareholders in cardiovascular disease enterprises: Part III: cellular and molecular clues to heart and arterial aging. Circulation 2003; 107: 490-7. 